\title{
The long secondary periods in semi-regular variables
}

\author{
P.R. Wood, A.E. Olivier \\ Research School of Astronomy 6 Astrophysics, Australian National \\ University, Cotter Road, Weston Creek ACT 2611, Australia \\ S.D. Kawaler \\ Department of Physics and Astronomy, Iowa State University, Ames IA \\ 50011, USA
}

\begin{abstract}
About $25 \%$ of variable asymptotic giant branch stars in the LMC have light curves that are modulated by a long secondary period of length $\sim 1-4 \mathrm{yr}$. Solar-vicinity analogs of these stars show radial velocity curves that are asymmetric and of small amplitude $\left(\sim 3-5 \mathrm{~km} \mathrm{~s}^{-1}\right)$. Possible explanations for the photometric and radial velocity variations include: eccentric motion of an orbiting companion of mass $\sim 0.1 \mathrm{M}_{\odot} ;$ radial and nonradial pulsation; rotation of an ellipsoidal-shaped red giant; episodic dust ejection; and star spot cycles. We find that there are severe difficulties with each of these models. Thus, the long secondary periods are the only unexplained type of large-amplitude stellar variability known at this time.
\end{abstract}

\section{Introduction}

Payne-Gaposhkin (1954) and Houk (1963) observed long ago that the light curves of some semi-regular (SR) variables in the solar vicinity show a long secondary period (LSP) of length typically $\sim 400-1500 \mathrm{~d}$, approximately ten times longer than the period of the primary pulsation period. Some examples of the light curves of these stars are given in Kiss et al. (1999). Recent observations of samples of local SR variables by Percy \& Bakos (2003) indicate that about $30 \%$ of SR variables exhibit LSPs.

MACHO observations of LMC red giants show that $\sim 25 \%$ of all variable AGB stars show long secondary periods roughly nine times longer than the primary period, which is typically $\sim 30-200 \mathrm{~d}$ (Wood et al. 1999). Some light curves of the LMC variables are shown in Wood et al. (1999). The LMC results show that both the short primary period and the long secondary period fall on one of the five distinct period-luminosity sequences exhibited by AGB variables (several papers in these proceedings show these sequences). Theoretical analysis shows that the shorter period belongs to a first or second overtone radial pulsation mode (Wood et al. 1999).

The origin of the longer secondary period is unclear. It can not be the radial fundamental pulsation mode since the long secondary period is $\sim 4$ times longer than the fundamental mode period. However, "strange" modes exist in 
red giants with periods similar to those of the LSPs and they could explain the LSPs (Wood 2000). A major problem with these modes is that they are extremely damped and they are unlikely to be seen in real stars. As an alternative explanation, Wood et al. (1999) suggested a binary model for the LSPs wherein the LSP results from obscuration of the red giant by a cloud of dust and gas associated with an orbiting companion.

If these stars are in binary systems, or are pulsating in a strange mode, they will exhibit radial velocity variations with a period equal to that of the long secondary period. Hinkle et al. (2000) have shown that small velocity variations of a few $\mathrm{km} \mathrm{s}^{-1}$ and with a period consistent with that of the LSP do indeed exist. We have found a similar result. Here, we combine the Hinkle et al. (2000) results with our own results in an attempt to determine the origin of the LSPs.

\section{Results for a typical SR variable with a long secondary period}

Fig. 1 shows various quantities plotted against time for the solar-vicinity SR variable ZEri (from Wood et al. 2003). Z Eri is known to have an LSP with a period of $\sim 800 \mathrm{~d}$. This radial velocity curve of $Z$ Eri is quite asymmetric, and is typical of the radial velocity curves in these stars. If the radial velocity variation is assumed to be due to radial pulsation, the velocity curve can be integrated to yield the radius change. The radius change will give rise to a change in $T_{\text {eff }}$ even without any change in luminosity, and this is shown by the solid line in Fig. 1 . The dotted line shows the change in $T_{\text {eff }}$ expected if an allowance for luminosity is included. A very distinct feature of the spectra of all the stars with LSPs examined so far is a strong variation with the LSP of the equivalent width of the $\mathrm{H} \alpha$ absorption line, as shown in the top panel of Fig. 1. Since the $\mathrm{H} \alpha$ line in these very cool stars must come from some sort of chromospheric layer (Luttermoser et al. 1994), this indicates that the source of chromospheric activity varies strongly with the LSP. Another spectral feature that varies markedly with the LSP is the NaD line profile. This suggests that the stellar wind is modulated by the LSP.

\section{Possible explanations for the long secondary periods}

Possible explanations for the long secondary periods include: radial or nonradial pulsation; binary companions; rotating ellipsoidal-shaped red giants; episodic dust formation; or star spot cycles. Here we summarize the discussion of these possibilities, as given in Wood et al. (2003).

Radial pulsation: This can be rejected since neither $T_{\text {eff }}$ (Fig. 1) nor the short period vary as predicted for the large radius change associated with the LSP. Nonradial pulsation: The lack of variation of $T_{\text {eff }}$ is also a problem for nonradial pulsation. Only the $g$ modes have the correct periods but they are evanescent throughout the convective envelope and should have negligible amplitudes at the stellar surface.

Binary companions: For typical observed periods and velocity curves, tidal forces should lead to orbital decay and merging in $\sim 10^{3} \mathrm{yr}$. Another problem with the binary model is that the existing well-determined radial velocity curves have a 


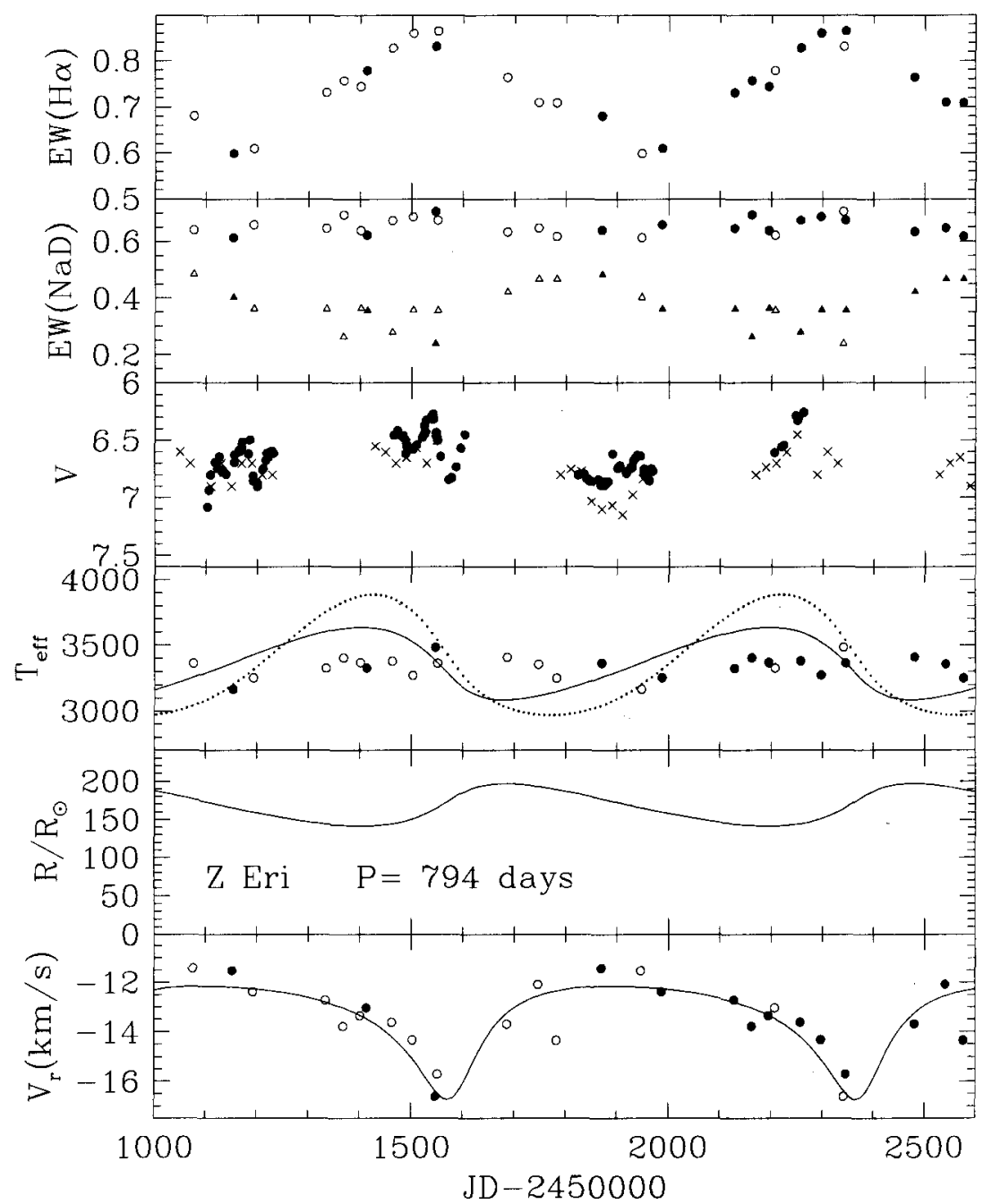

Figure 1. Various properties of Z Eri plotted against Julian Date. Solid symbols are values plotted at the date of measurement while open symbols are values that have been shifted forward or backwards by one period of $794 \mathrm{~d}$. From the top: the equivalent width of the $\mathrm{H} \alpha$ absorption line $(\AA)$; the average equivalent width of the two $\mathrm{NaD}$ absorption lines in $\AA$ (circles) and the height of the central emission peak relative to the continuum (triangles); the $V$ light curve obtained from the AAVSO $(\bullet)$ and from the AFOEV database $(x$, shifted by +0.2 mag to match the AAVSO magnitudes); the effective temperature $T_{\text {eff }}$ obtained from the strength of the TiO band at $7050 \AA$ (circles), with curves indicating the expected change in $T_{\text {eff }}$ if the LSP is due to radial pulsation - see text for details; the radius variation expected if the radial velocity variation is due to radial pulsation; and the heliocentric observed velocity, together with a binary fit to the velocities. 
characteristic shape. If these were really binary systems, the chance that the angle of periastron would be aligned as required is only $\sim 7 \%$.

Rotating prolate spheroids: If the LSP stars are rotating prolate spheroids, they will give rise to the characteristic velocity curves. However, it is not obvious why the stars would lie on a period-luminosity sequence and the rotation velocities required in this model seems somewhat too high.

Episodic dust ejection: Episodic dust ejection by the dust $\kappa$-mechanism (Winters et al. 1994; Höfner et al. 1995) could explain the LSP light curves. However, this is a near-circumstellar phenomenon and it is not clear why the much deeper photospheric layers should show radial velocity variations at the period of the LSP. Furthermore, there is no evidence for circumstellar dust around the stars having LSPs (Hinkle et al. 2002; Olivier \& Wood 2003).

Star spot cycles: Chromospheric activity is clearly associated with the LSPs, possibly due to magnetic activity. However, this does not explain the radial velocity variations. Color variations of the $\mathrm{LMC}$ variables are inconsistent with rotating dark spots.

\section{Conclusions}

At the present time, there is clearly no satisfactory explanation for the LSPs in red semi-regular variables. To our knowledge, these are the only large amplitude, (semi-)periodic stellar variations which are currently unexplained. Given that $\sim 25-30 \%$ of AGB variables show LSPs, we speculate that the mechanism which produces the LSPs is also the mechanism responsible for the production of asymmetric planetary nebulae.

\section{References}

Hinkle K.H., Lebzelter T., Joyce R.R., Fekel F.C. 2002, AJ, 123, 1002

Höfner S., Feuchtinger M.U., Dorfi E.A. 1995, A\&A, 297, 815

Houk N. 1963, AJ, 68, 253

Kiss L.L., Szatmáry K., Cadmus R.R. Jr, Mattei J.A. 1999, A\&A, 346, 542

Luttermoser D.G., Johnson H.R. Eaton J. 1994, ApJ, 422, 351

Olivier E.A., Wood P.R. 2003, ApJ, 584, 1035

Payne-Gaposhkin C. 1954, Harvard Annals, 113, No. 4

Percy J.R., Bakos G.A. 2003, preprint

Winters J.M., Fleischer A.J., Gauger A., Sedlmayr E. 1994, A\&A, 290, 623

Wood P.R. and the MACHO COLLABORATION 1999, in IAU Symposium 191, AGB Stars, eds. T. Le Bertre, A. Lèbre \& C. Waelkens (San Francisco, ASP), 151

Wood P.R. 2000, in IAU Coll. 176, ASP Conf. Ser. Vol. 203, The Impact of Large-Scale Surveys on Pulsating Star Research, eds L. Szabados \& D. Kurtz (San Francisco, ASP), 379

Wood P.R., Olivier E.A., Kawaler S. 2003, ApJ, submitted 


\section{Discussion}

Feast: How certain are you that the galactic stars you have studied belong to your sequence $\mathrm{D}$ ?

Wood: Light curves for some of the local stars have been computed by Kiss et al. from amateur observations. These light curves are very similar to the light curves of the LMC stars observed by MACHO, indicating that the local and LMC stars are similar.

Pollard: RV Tauri stars of type RVb show numerous similarities to these stars: long-term light variations, sometimes colour variations, and exactly the same sort of radial velocity variations, although RV Tauri stars are slightly hotter. RVb stars show enhanced $\mathrm{H} \alpha$ emission at long-term minimum - could this be a clue as to cause of the long-term variations?

Wood: Yes, I'm sure there is something staring us in the face with these stars.

Shibahashi: I agree with that g-modes have large amplitudes only in the radiative core as far as the oscillations are eigenmodes. However, in the case of tidally forced oscillations, the amplitudes can be larger in the outer convective envelope than in the deep radiative core, even though the frequency range of the tidally forced oscillations is as low as that of the g-modes.

Wood: This model still requires a binary companion to drive oscillations, and having $25-30 \%$ of AGB stars with a close companion is unlikely.

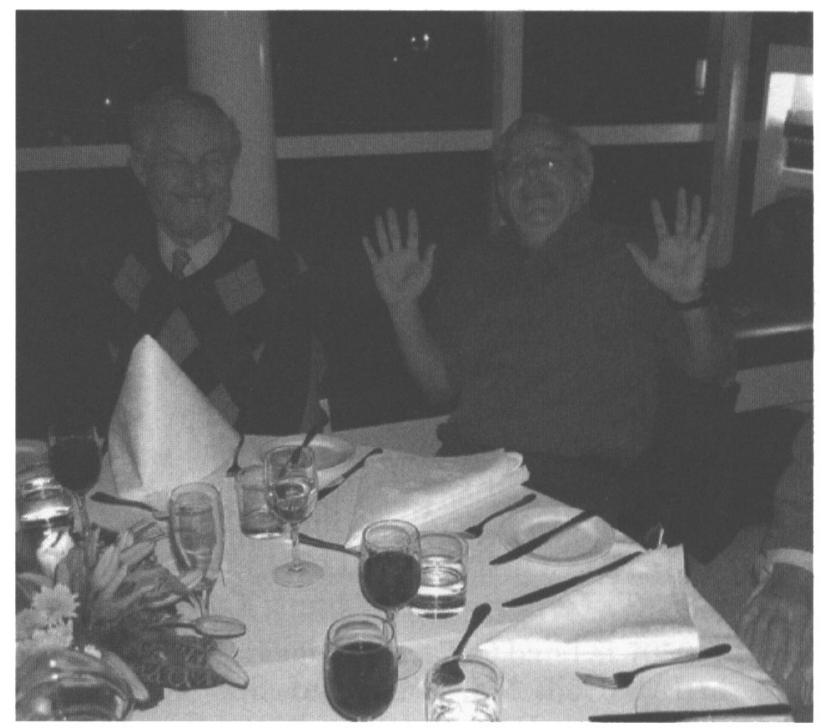

Tom Lloyd Evans and Peter Wood 